\title{
Thin accretion disc with a corona in a central magnetic field
}

\author{
Fazeleh Khajenabi ${ }^{1}$ - Mohsen Shadmehri ${ }^{2,3}$ • \\ Sami $\mathrm{Dib}^{4,5}$
}

(C) Springer-Verlag $\bullet \bullet \bullet \bullet$

\begin{abstract}
We study the steady-state structure of an accretion disc with a corona surrounding a central, rotating, magnetized star. We assume that the magnetorotational instability is the dominant mechanism of angular momentum transport inside the disc and is responsible for producing magnetic tubes above the disc. In our model, a fraction of the dissipated energy inside the disc is transported to the corona via these magnetic tubes. This energy exchange from the disc to the corona which depends on the disc physical properties is modified because of the magnetic interaction between the stellar magnetic field and the accretion disc. According to our fully analytical solutions for such a system, the existence of a corona not only increases the surface density but reduces the temperature of the accretion disc. Also, the presence of a corona enhances the ratio of gas pressure to the total pressure. Our solutions show that when the strength of the magnetic field of the central neutron star is large or the star is rotating fast enough, profiles of the physical variables of the disc significantly modify due to the existence of a corona.
\end{abstract}

Fazeleh Khajenabi

Mohsen Shadmehri

fazeleh.khajenabi@ucd.ie, mohsen.shadmehri@dcu.ie

Sami Dib

sami.dib@cea.fr

${ }^{1}$ School of Mathematical Sciences, University College Dublin, Belfield, Dublin 4, Ireland

${ }^{2}$ School of Mathematical Sciences, Dublin City University, Glasnevin, Dublin 9, Ireland

${ }^{3}$ Department of Physics, School of Science, Ferdowsi University, Mashhad, Iran

${ }^{4}$ Lebanese University, Faculty of Sciences, Department of Physics, El Hadath, Beirut, Lebanon

${ }^{5}$ Service d'Astrophysique, DSM/IRFU/SAp, CEA/Saclay, 91191, Gif-sur-Yvette Cedex, France
Keywords accretion discs - stars: magnetic field stars: neutron

\section{Introduction}

Accretion discs are believed to be present in a wide variety of astronomical systems. The standard theory of geometrically thin accretion discs has provided successful framework for understanding the basic physical properties of these objects (Shakura \& Sunyaev 1973). A solution for the structure of an accretion disc in an external magnetic field is desirable because accretion discs are frequently found around magnetic stars (Verbunt 1993; Warner 1995; Romanova et al 2003). The stellar magnetic fields interacts with the disc and can allow the transfer of angular momentum between the disc and the star and this significantly affects the structure of the disc and the spin evolution of the star (e.g. Ghosh \& Lamp 1978; Aly 1980; Wang 1987; Armitage, Clarke \& Tout 1999; Schenker et al 2002; Dai \& Li 2006). Matthews et al (2005) reformulated the standard disc solution to incorporate the effect of a torque from a stellar magnetic field. They studied the effects of varying the mass transfer rate, spin period and strength of the stellar magnetic field using their analytical solutions. Also, there are several simulations of the interactions between the stellar magnetic filed and accretion disc (e.g. Miller \& Stone 1997; Romanova et al 2002; Romanova et al 2003). Miller \& Stone (1997) investigated disc-star interaction for different geometries and stellar magnetic fields. Romanova et al (2002) described in detail the disc-star interaction for the case of slowly rotating stars and also cases of fast rotating stars.

On the other hand, there are varying models of a hot corona above a cool disc (e.g. Haardt\& Maraschi 1993). Begelman, McKee \& Shields (1983) presented detailed calculations of the formation of the corona by 
irradiation from the central source. Rozanska \& Czerny (1996) and Rozanska et al (1999) investigated the effect of illumination by the central source on the accretion disc for either a black hole as in AGN or a neutron star as in X-ray binary sources. It was found that the disc and the corona are separated by a boundary layer or heated upper region of the disc, i.e. the region above the disc becomes stratified with increasing temperature and decreasing density (see also Rozanska et al 2002). Jimenez-Garate, Raymond \& Liedahi (2002) calculated the vertical structure of an Accretion Disc Corona (ADC) and allowed the radius of the corona to extend to the same radius of the accretion disc. Low Mass X-ray Binaries (LMXBs) have two continuum components of radiation: simple blackbody emission from the neutron star plus comptonized emission from an extended corona above accretion disc (e.g. Church \& Balucinska-Church 1995). Church \& Balucinska (2004) have demonstrated the importance of the size of the ADC to the correct description of Comptonization and they derived the comptonized spectrum of an LMXB based on the thermal Comptonization of seed photons. They estimated the radial extension of ADC in some LMXBs up to $10^{9} \mathrm{~cm}$.

Generally, models for generating an ADC can be divided into two groups: (1) The dissipation of acoustic or magnetic energy flux generated by convective or viscous turbulence within the disc (e.g. Galeev, Rosner \& Vaiana 1979; Merloni \& Fabian 2002; Merloni 2003); or (2) the evaporation of disc material by the central source (e.g. Begelman, McKee \& Shields 1983).

Differential rotation of a standard accretion disc amplifies the magnetic field. This may result in a magnetic active corona surrounding the disc (Burm 1986). These magnetic loops which thread the disc are analogous to the solar case. The problem of magnetic flux escape from an accretion disc has been studied by many authors (Stella \& Rosner 1984; Heyvaerts \& Priest 1989; Shibata, Tajima \& Matsumoto 1990; Romanova et al 1998).

The are reasons to believe that some parts of the ADC may find itself embedded in the field of the central object. Stollman \& Kuperus (1988) assumed that the neutron stars in some of the LMXB have magnetic fields, which are capable of holding the accretion disc at some distance from the stars surface. They developed a model for the interaction of the magnetic field of the neutron star and the magnetic loops in the corona above the disc. Flaring interactions between accretion disc and neutron star magnetosphere has also been studied by many authors (e.g. Aly \& Kuijpers 1990). Magnetic loops of the accretion disc which extend toward corona may contribute to angular momentum transport in the disc (Burm \& Kuperus 1988). However, the possible effects of the corona on the structure of a thin accretion disc under the influence of a stellar magnetic field have not been studied to our knowledge. In this paper, we extended Matthews et al (2005) analysis by considering the corona of the disc as well. The following question is addressed: what is the effect of the corona on the steady state structure of a magnetically torqued thin accretion disc? We obtain a set of analytical solutions for a thin accretion disc with a corona, to which a magnetic torque due to the central object (e.g. neutron star) is applied. We perform a parameter study to explore how physical variables of the disc such as density or temperature may be modified due to changes in stellar magnetic field or stellar spin. In section 2, we discuss the basic assumptions and present the main equations of the model. Typical physical properties of the solutions will be studied in section 3 . In the final section we conclude the paper by a summary of our results.

\section{General formulation}

We assume that all angular momentum transport takes place in the disc and the mass accretion rate $\dot{M}$ is constant with radius and time. However, the microphysics mechanism of the angular momentum transfer remains unknown. Shakura \& Sunyaev (1973) replaced all the missing physics by a parameter $\alpha$. This approach has been widely used for studying the dynamics and structure of accretion flow. A promising mechanism for driving the turbulence responsible for angular momentum and energy transport is the action of the magneto-rotational instability (MRI) that is expected to take place in such discs (Balbus \& Hawley 1991). But for a disc-corona system, it is typically thought that the viscous stress, assumed to be magnetic in nature, transports angular momentum and initially randomise the gravitational binding energy near the midplane. The magnetized fluid elements, which are buoyant with respect to their surroundings, dissipate above the disc. Merloni \& Fabian (2002) and Merloni (2003) presented very detailed discussions about dissipation in the corona and its relation with angular momentum transport in the disc itself. Our disc-corona model is developed along the line proposed by Merloni \& Fabian (2002) and Merloni (2003).

We consider a more general prescription for the viscous stresses $\tau_{\mathrm{r} \phi}$ (Taam \& Lin 1984; Watarai \& Mineshige 2003; Merloni \& Nayakshin 2006):

$\tau_{\mathrm{r} \phi}=-\alpha_{0} p^{1-\mu / 2} p_{\text {gas }}^{\mu / 2}$, 
where $\alpha_{0}$ and $0 \leq \mu \leq 2$ are constants. Also, $p$ is the sum of the gas and radiation pressures. Phenomenological models generally assume that at each radius, a fraction $f$ of accretion energy is released in the reconnected magnetic corona. Assuming that turbulence inside the disc is MRI-driven, such a fraction $f$ of the binding energy is transported from large to small depths by Poynting flux, Merloni \& Nayakshin (2006) estimated the fraction $f$ as

$f=\sqrt{2 \alpha_{0} \beta^{\mu / 2}}$

where $\beta$ is the ratio of gas pressure to the total pressure. So, in this model, this fraction $f$ is not a free parameter. Actually, it is very difficult to present a detailed analytical model which gives us the fraction $f$ as a function of the disc physical variables structure and MRI (even without central magnetic torque). We appreciate on possible effect of central magnetic torque on energy exchange $f$. But it is beyond our study because of severe limitations of our phenomenological approach. But we would like to emphasize that the amount of the energy exchange corresponding to solutions with central magnetic torque (our solutions) and without central torque is not the same. Because for solutions with a central magnetic torque, we have an extra magnetic torque in angular momentum equation (see equation (17) which dramatically changes the solutions comparing to the solutions without that term (see solutions of Matthews et al. (2005)). In fact, equation (2) prescription is based on MRI inside of the disc, irrespective of the (non)existence of external magnetic torque. So, with central magnetic torque its effect on the energy exchange appears by changing disc structure (through an extra magnetic term in angular momentum equation).

The rotation curve is dominated by a Newtonian point mass $M$, as relativistic effects are only important at small radii. Thus, the rotational angular velocity of the disc is Keplerian, i.e. $\Omega_{\mathrm{K}}=\sqrt{G M / R^{3}}$. Having equation (11) as a prescription for the viscous stresses and equation (2) as an expression for the fraction of power dissipated in the corona, we can write the basic equations of the disc. Vertical hydrostatic equilibrium of the disc implies

$\frac{p}{\Sigma}=\frac{\Omega_{\mathrm{K}}^{2} H}{2}$.

In order to write the azimuthal component of equation of motion, the specific magnetic torque of the central neutron star can be written as

$\Lambda=\frac{\sqrt{G M} R^{1 / 2}}{t_{\Lambda}}$, where $t_{\Lambda}$ is the time-scale on which the local disc material gains angular momentum. This torque appears because of the interaction of the field lines of the central, rotating magnetized neutron star with its surrounding partially ionized inner accretion disc. Actually neither the physics of angular momentum transport by the magnetic field of the central star, nor the mechanism of penetration of the magnetic field into the accretion disc are well understood. Nevertheless we can present some physical prescriptions for the magnetic torque of the central neutron star. Actually, there are some interactions between the magnetic tubes above the disc and the magnetic field of the neutron star. However, we concentrate on the steady structure of the disc and such complicated interactions are neglected in our model. Formation of the corona is a direct consequence of the magnetic tubes of the accretion disc. On the other hand, the physical properties of the accretion disc are modified because of the existence of the magnetic torque and the dissipated energy into the corona depends on the structure of the disc. Thus, the effect of the stellar magnetic field on the magnetic tubes is included via its torque on the disc indirectly in our model. Figure 1 show schematically the magnetic field of the neutron star and the magnetic tubes of the disc. The magnetic structures in the corona of the disc should be of a small scale, because turbulent eddies in the disc are believed to have the disc thickness as a characteristic size, as should the magnetic loops which pop out as a result of magnetic buoyancy. However, there is a possibility of forming larger magnetic loops above the disc by reconnecting small scale loops (e.g., Heyvaerts $\&$ Priest 1984). Analysis of such complexities is beyond this paper.

In equation (4), the torque time-scale $t_{\Lambda}$ is equivalent to the magnetic time-scale $t_{\text {mag }}$. Magnetic field of

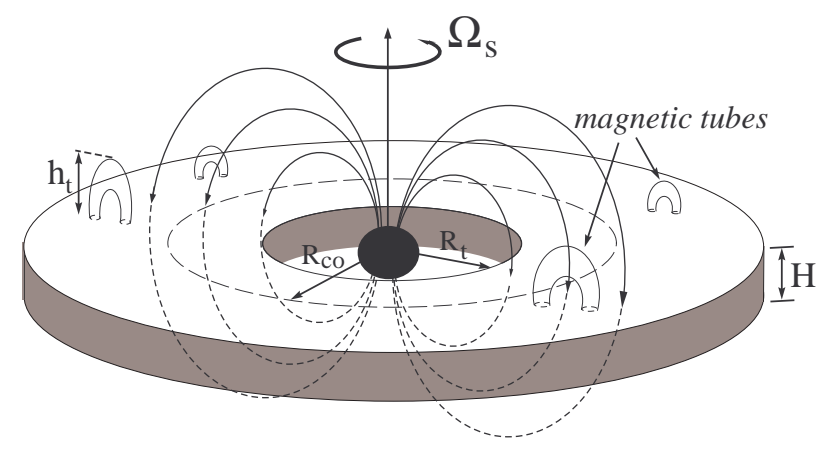

Fig. 1 Schematic of the magnetic field of the neutron star and magnetic tubes of the accretion disc. The truncation and the corotation radii are marked by $R_{\mathrm{t}}$ and $R_{\mathrm{co}}$, respectively. 
the neutron star has a dipolar form, i.e. $B_{\mathrm{z}} \sim|\mu| R^{-3}$ where $\mu$ is the magnetic moment of the star. Depending on the magnetic interaction model, the magnetic time-scale can be parametrized in the form (Livio \& Pringle 1992; Campbell \& Heptinstall 1998; Matthews et al 2005)

$t_{\mathrm{mag}}=\frac{2 \Sigma}{\beta_{M}} \frac{R^{\gamma+2}}{\left[\left(R / R_{c o}\right)^{3 / 2}-1\right]}$,

where $\gamma$ is determined by the magnetic interaction model (e.g., Campbell \& Heptinstall 1998) and $\beta_{M}$ is calculated by the mass and the magnetic moment of the star,

$\beta_{M}=\frac{|\mu|^{2}}{2 \pi \sqrt{G M}}$.

In our fully magnetized case we have $\gamma=7 / 2$ (Livio \& Pringle 1992; Matthews et al 2005). Also, in the above equation, the corotation radius is represented by $R_{c o}$ which is by definition the radius of the accretion disc at which, the star and the disc rotate at the same angular rate (Figure 1). Note that the dipole and spin axes are taken to be parallel to $z$, and the whole system is assumed to be axisymmetric.

Now, we can write the azimuthal component of the equation of motion as

$\Sigma v_{\mathrm{R}} \frac{d}{d R}\left(R v_{\varphi}\right)=\frac{1}{R} \frac{d}{d R}\left(R^{2} \tau_{r \phi}\right)+\Sigma \Lambda$,

where $v_{\mathrm{R}}$ and $v_{\varphi}$ are the radial and the rotational velocities of the disc. We note that the disc is rotating with Keplerian profile, i.e. $v_{\varphi}=v_{\mathrm{K}}$. The $r-\varphi$ component of viscous stress tensor is prescribed by equation (11). Also, the parameter $\Lambda$ is representing the specific torque of the central neutron star which is given by equation (44). After mathematical manipulations, we can write equation (7) as

$8 \pi \alpha_{0} H\left(p_{\text {gas }}\right)^{\mu / 2} p^{(2-\mu) / 2}=3 \Omega_{\mathrm{K}} \dot{M} q^{4} v$

where,

$v=1-\frac{\beta_{M}}{\dot{M}} \frac{\pi h}{R^{\gamma}(\gamma-2) q^{4}}$,

$h=\left(\frac{R}{R_{c o}}\right)^{\frac{3}{2}}\left[1-\left(\frac{R_{t}}{R}\right)^{2-\gamma}\right]-\frac{\gamma-2}{\gamma-\left(\frac{1}{2}\right)}\left[1-\left(\frac{R_{t}}{R}\right)^{\frac{1}{2}-\gamma}\right]$,

$q=\left[1-\left(\frac{R_{t}}{R}\right)^{\frac{1}{2}}\right]^{\frac{1}{4}}$.

The truncation radius $R_{t}$ denotes the inner boundary of the accretion disc. A common feature of all magnetic star-disc interaction models is that the stellar magnetosphere is strong enough to disrupt the accretion disc at some radius $R_{t}$ above the surface of the star. There are several methods to locate the truncation radius. We use the approach of Matthews et al (2005). They presented an approximate relation for $\gamma=7 / 2$ as

$\frac{R_{t}}{R_{c o}} \simeq\left[\frac{Q}{2}\left(\sqrt{\frac{4}{Q}+1}-1\right)\right]^{2 / 3}$,

where the nondimensional parameter $Q$ is determined by the ratio $\beta_{M}$, accretion rate and the corotation radius, i.e.

$Q=2 \pi R_{c o}^{-\gamma}\left(\frac{\beta_{M}}{\dot{M}}\right)$.

The truncation and corotation radii are shown schematically in Figure 1

The energy equations is given by

$\sigma T_{\mathrm{eff}}^{4}=\frac{3}{8 \pi} \Omega_{\mathrm{K}}^{2} \dot{M} q^{4} v(1-f)$,

where as we discussed $f=\sqrt{2 \alpha_{0} \beta^{\mu / 2}}$. Also, we can assume that the vertical transport of heat is by radiative diffusion which implies the midplane and the surface temperatures are related by

$T=\left(\frac{3}{8} \kappa \Sigma\right)^{1 / 4} T_{\text {eff }}$,

where $\kappa$ is the opacity coefficient. In order to limit the parameter space in our analysis, we simply assume the electron opacity for this coefficient, i.e. $\kappa=\kappa_{e}=0.4$ $\mathrm{cm}^{2} \mathrm{~g}^{-1}$.

Now, equations (3), (8) and (11) are the main equations which enable us to find $p$ and $T$ as functions of $R$ and $\beta$ and the other input parameters. Thus,

$$
\begin{aligned}
& T=\left(\frac{4 \sigma \Omega_{\mathrm{K}}}{3 \kappa \alpha_{0}}\right)^{-1 / 2}\left(\frac{16 \pi^{2} \alpha_{0}^{2} c k_{\mathrm{B}}}{3 \sigma \mu_{\mathrm{m}} m_{\mathrm{H}} \dot{M}^{2} \Omega_{\mathrm{K}}^{4} q^{8} v^{2}}\right)^{-1 / 3} \\
& \times \frac{\left(1-\sqrt{2 \alpha_{0} \beta^{\mu / 2}}\right)^{1 / 2}}{(1-\beta)^{1 / 3}} \beta^{(4-\mu) / 12}, \\
& p=\left(\frac{4 \sigma \Omega_{\mathrm{K}}}{3 \kappa \alpha_{0}}\right)^{-1 / 2}\left(\frac{16 \pi^{2} \alpha_{0}^{2} c k_{\mathrm{B}}}{3 \sigma \mu_{\mathrm{m}} m_{\mathrm{H}} \dot{M}^{2} \Omega_{\mathrm{K}}^{4} q^{8} v^{2}}\right)^{-2 / 3} \\
& \times \frac{\left(1-\sqrt{2 \alpha_{0} \beta^{\mu / 2}}\right)^{1 / 2}}{(1-\beta)^{2 / 3}} \beta^{(8-5 \mu) / 12} .
\end{aligned}
$$

There is an algebraic equation for $\beta$ as follows

$$
\frac{k_{\mathrm{B}}}{\mu_{\mathrm{m}} m_{\mathrm{H}}}\left(\frac{4 \sigma \Omega_{\mathrm{K}}}{3 \kappa \alpha_{0}}\right)^{-3 / 2}\left(\frac{16 \pi^{2} \alpha_{0}^{2} c k_{\mathrm{B}}}{3 \sigma \mu_{\mathrm{m}} m_{\mathrm{H}} \dot{M}^{2} \Omega_{\mathrm{K}}^{4} q^{8} v^{2}}\right)^{-5 / 3}
$$



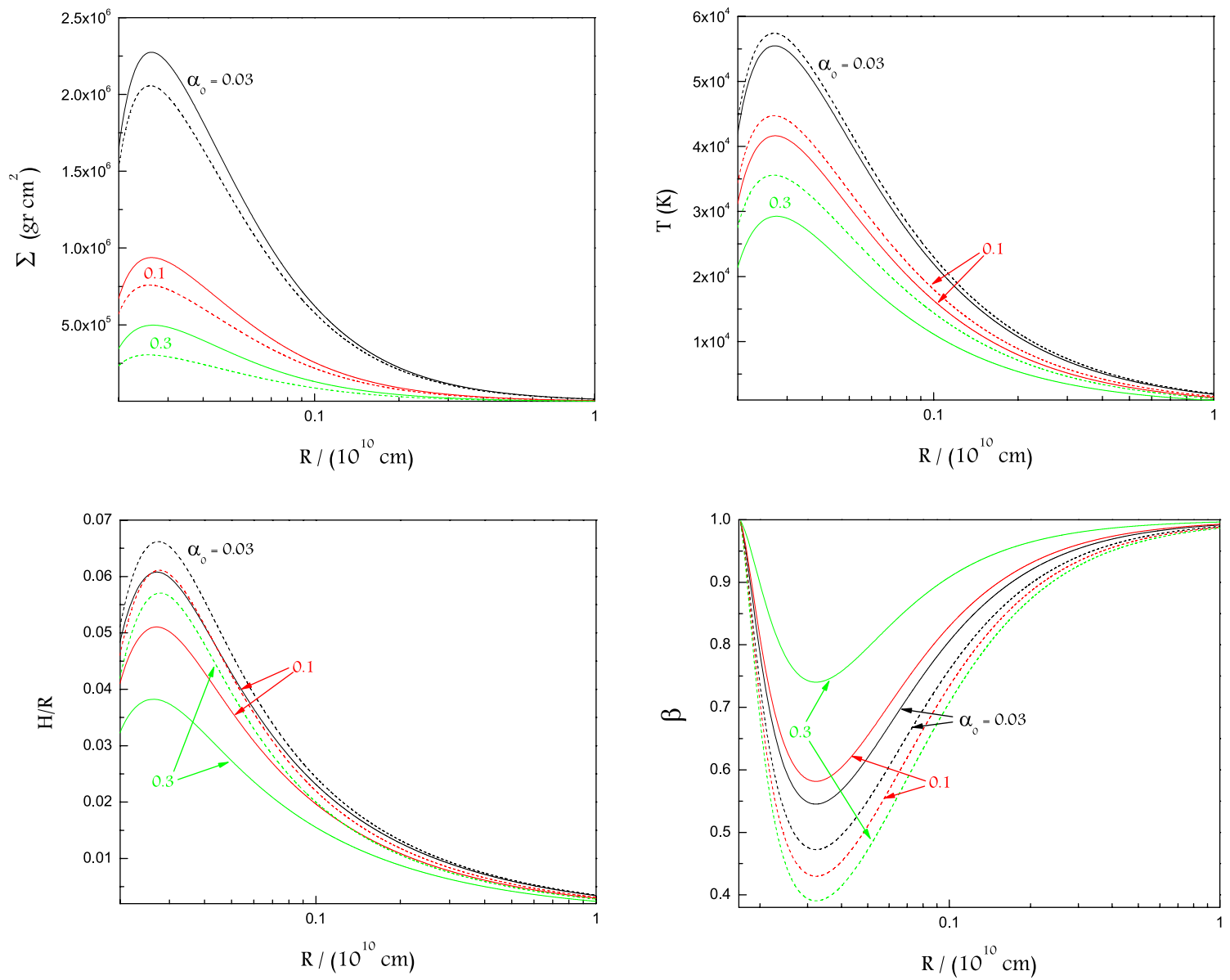

Fig. 2 Profiles of the physical variables for a disc with a corona (solid lines) and without a corona (dashed lines) vs. radial location in the disc with $\mu=1.0, \mu_{\mathrm{m}}=0.6, \hat{k}=1, M_{1}=1.4, \dot{M}_{16}=1, P_{\text {spin }}=1 \mathrm{~s}$ and $B_{s}=10^{12}$ G. Each curve is labeled by its corresponding viscosity coefficient $\alpha_{0}$. Also, in the online version of the paper, curves are shown by different colors depending on the viscosity coefficient. The top left-hand and right-hand plots show the surface density and the temperature of the accretion disc, respectively. The ratio of the thickness of the disc and the radius is shown in the bottom left-hand plot. Finally, the bottom right-hand plot shows profile of the ratio $\beta$ of gas pressure to total pressure. Generally, we see that the corona becomes more effective when the viscosity coefficient $\alpha_{0}$ has larger values. 

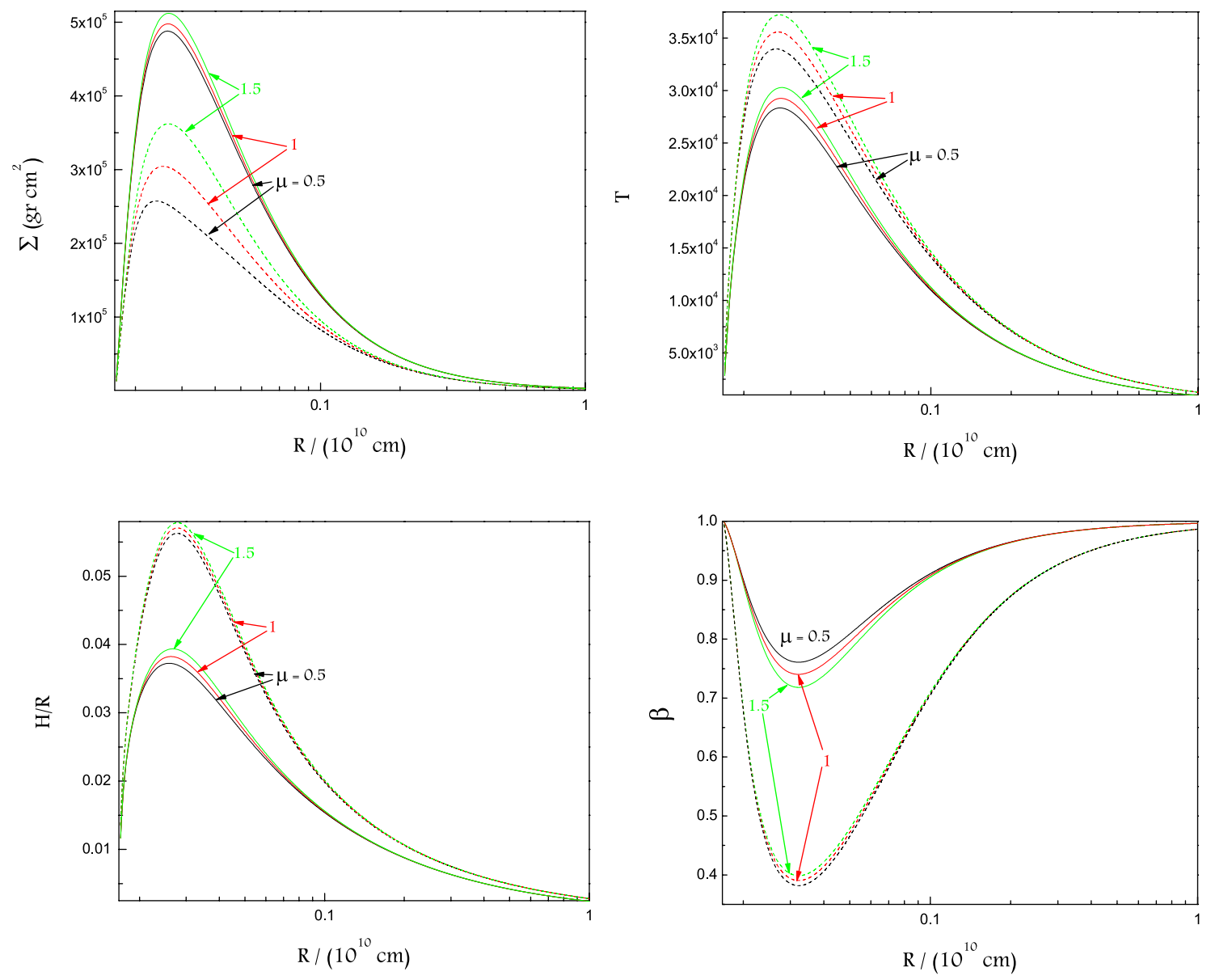

Fig. 3 Profiles of the physical variables for a disc without a corona (dashed lines) and with a corona (solid lines) vs. radial location in the disc with $\alpha_{0}=0.3, \mu_{\mathrm{m}}=0.6, \hat{k}=1, M_{1}=1.4, \dot{M}_{16}=1, P_{\text {spin }}=1 \mathrm{~s}$ and $B_{s}=10^{12} \mathrm{G}$. These plots show dependence of the physical variables to the viscosity exponent $\mu$. Typical behaviors of the ratio $H / R$ and the ratio of the gas pressure to the total pressure, $\beta$, for the case without a corona are not very sensitive to the exact value of the viscosity exponent. But in the case of nonexistence of a corona, profiles of the surface density and the temperature of the accretion disc are not independent of the parameter $\mu$. As this exponent increases, both the surface density and the temperature increase. 

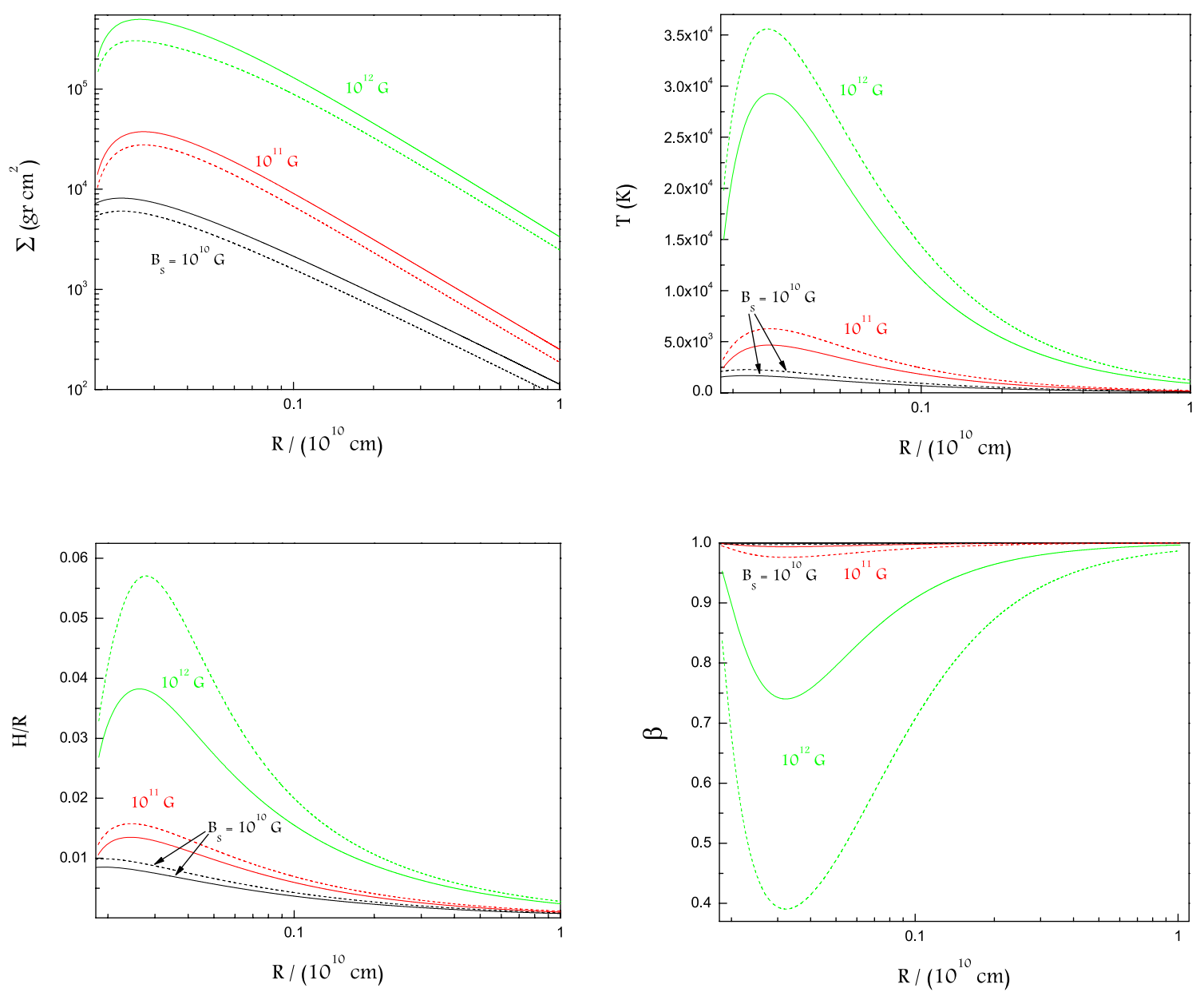

Fig. 4 Profiles of the physical variables for a disc without a corona (dashed lines) and with a corona (solid lines) vs. radial location in the disc with $\alpha_{0}=0.3, \mu=1.0, \mu_{\mathrm{m}}=0.6, \hat{k}=1, M_{1}=1.4, \dot{M}_{16}=1$ and $P_{\text {spin }}=1$ s. Effects of the magnetic field of the central neutron star are shown in these plots. Each curve is labeled by the magnetic field at the surface of the neutron star, i.e. $B_{s}$. As the magnetic field increases, the possible differences between the solutions with a corona and without a corona become more significant. The bottom right-hand plot shows profile of the ratio $\beta$ of gas pressure to total pressure. 

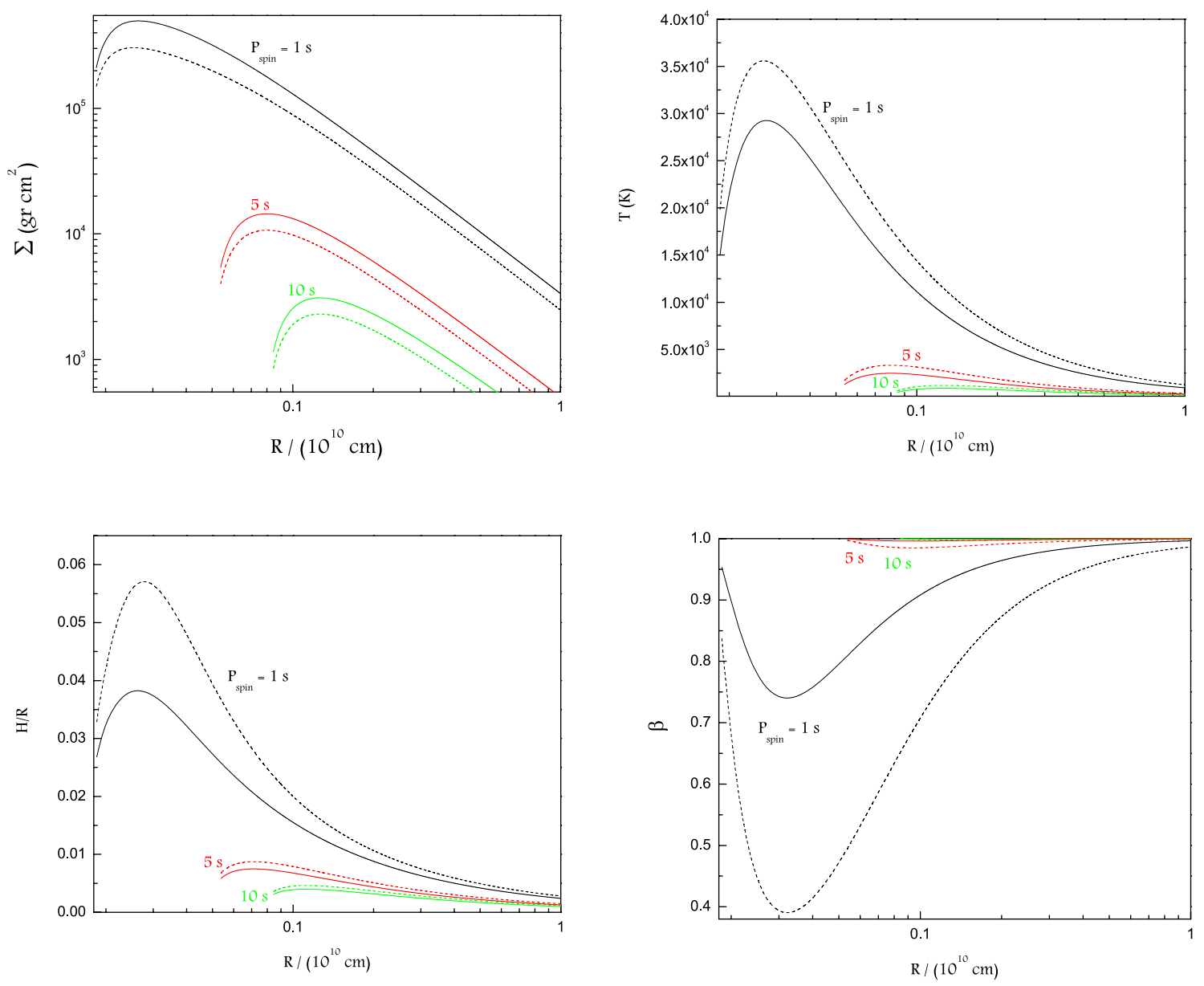

Fig. 5 Profiles of the physical variables for a disc without a corona (dashed lines) and with a corona (solid lines) vs. radial location in the disc with $\alpha_{0}=0.3, \mu=1.0, \mu_{\mathrm{m}}=0.6, \hat{k}=1, M_{1}=1.4, \dot{M}_{16}=1$ and $B_{s}=10^{12} \mathrm{G}$. The bottom right-hand plot shows profile of the ratio $\beta$ of gas pressure to total pressure. The central magnetic neutron star is rotating at different speed and each curve is labeled by the rotational period of the star. We see that the truncation radius increases as the central neutron star is rotating slower. 
$\times\left(\frac{8 \pi \alpha_{0}}{3 \Omega_{\mathrm{K}}^{2} \dot{M} q^{4} v}\right)^{2} \frac{\left(1-\sqrt{2 \alpha_{0} \beta^{\mu / 2}}\right)^{3 / 2}}{(1-\beta)^{5 / 3}} \beta^{(8+\mu) / 12}=1$,

where $\mu_{\mathrm{m}}$ is the mean particle mass in units of the hydrogen atom mass, $m_{\mathrm{H}}$. The other constants have their usual meanings.

In order to study the behavior of our solutions, it is more convenient to introduce dimensionless variables. For the central mass $M$, we introduce $M_{1}=M / M_{\odot}$ and for opacity we assume $\hat{\kappa}=\kappa / \kappa_{e}$. The radial distance from the star, in the plane of the disc, is represented by $R$ in units of $10^{10} \mathrm{~cm}$. So, we introduce $r_{10}=R /\left(10^{10} \mathrm{~cm}\right)$. Also $\dot{M}_{16}=\dot{M} /\left(10^{16} \mathrm{gs}^{-1}\right)$ represents the mass accretion rate in units of $10^{16} \mathrm{~g} \mathrm{~s}^{-1}$.

We can rewrite our solutions as

$$
\begin{aligned}
& \rho=2.63 \times 10^{-10} \alpha_{0}^{-1 / 2} \hat{\kappa}^{3 / 2} M_{1}^{5 / 4} \dot{M}_{16}^{2} q^{8} v^{2} r_{10}^{-15 / 4} \\
& \times \beta^{(8-\mu) / 4}(1-\beta)^{-2}\left(1-\sqrt{2 \alpha_{0} \beta^{\mu / 2}}\right)^{3 / 2}, \\
& p=40.47 \times \alpha_{0}^{-5 / 6} \hat{\kappa}^{1 / 2} M_{1}^{13 / 12} \dot{M}_{16}^{4 / 3} q^{16 / 3} v^{4 / 3} r_{10}^{-13 / 4} \\
& \times \beta^{(8-5 \mu) / 12}(1-\beta)^{-2 / 3}\left(1-\sqrt{2 \alpha_{0} \beta^{\mu / 2}}\right)^{1 / 2}, \\
& \frac{H}{R}=0.24 \times \alpha_{0}^{-1 / 6} \hat{\kappa}^{-1 / 2} M_{1}^{1 / 6} \dot{M}_{16}^{-1 / 3} q^{-4 / 3} v^{-1 / 3} \\
& \times \beta^{-(8+\mu) / 12}(1-\beta)^{2 / 3}\left(1-\sqrt{2 \alpha_{0} \beta^{\mu / 2}}\right)^{-1 / 2},
\end{aligned}
$$

and the ratio $\beta$ is obtained from the non-dimensional form of equation (15), that is,

$$
\begin{aligned}
& 4.69 \times 10^{-7} \alpha_{0}^{1 / 6} \hat{\kappa}^{3 / 2} M_{1}^{7 / 12} \dot{M}_{16}^{4 / 3} q^{16 / 3} v^{4 / 3} r_{10}^{-7 / 4} \\
& \times \beta^{(8+\mu) / 12}(1-\beta)^{5 / 3}\left(1-\sqrt{2 \alpha_{0} \beta^{\mu / 2}}\right)^{3 / 2}=1 .
\end{aligned}
$$

We can also calculate the surface density as

$$
\begin{aligned}
& \Sigma=2.12 \times 10^{-4} \alpha_{0}^{-2 / 3} \hat{\kappa} M_{1}^{17 / 12} \dot{M}_{16}^{5 / 3} q^{20 / 3} v^{5 / 3} r_{10}^{-11 / 4} \\
& \times \beta^{(4-\mu) / 3}(1-\beta)^{-4 / 3}\left(1-\sqrt{2 \alpha_{0} \beta^{\mu / 2}}\right)^{1 / 2} .
\end{aligned}
$$

Typical disc structure will now be illustrated, using the full disc solutions quoted in equations (16), (17) and (18). However, the physical variables depend not only on the radial distance but also on the ratio of the gas pressure to the total pressure which can be calculated at each radius from algebraic equation (19). Having analytical solutions, we can study physical properties of our disc and corona system. In the next section, we will do a parameter study of our solutions.

\section{Analysis}

We fix the central mass, the mass transfer rate, the opacity and the mean molecular weight, respectively as $M_{1}=1.4, \dot{M}_{16}=1, \hat{\kappa}=1$ and $\mu_{m}=0.6$. The other input parameters are changed to illustrate their possible effects on the physical properties of the system. Typical behaviors of the solutions for disc with a corona (solid curves) and without a corona (dashed curves) are shown in all subsequent figures. Each curve is labeled by its corresponding parameter, though color versions are available in the online version of the paper. Note that the radial extension of the plots are up to $10^{10} \mathrm{~cm}$, significant effects of the corona and the central magnetic field are seen at radii less than $10^{9} \mathrm{~cm}$, generally.

Figure 2 shows typical behavior of the physical quantities of the accretion disc when the viscosity coefficient $\alpha_{0}$ changes from low value 0.03 to high value 0.3 . The profile of the surface density is shown in the top lefthand plot of Figure2, When the viscosity coefficient increases, the surface density decreases both in the cases with and without a corona. This behavior can be explained simply by the fact the the accretion rate is constant in our model. So, the radial velocity (which is proportion to viscosity coefficient) is inversely proportional to the surface density. Thus, when the viscosity coefficient increases, the radial velocity increases too. But the surface density decreases with the viscosity coefficient. However, the corona significantly increases the surface density. The top right-hand plot of Figure 2 shows the presence of a corona serves to cool the disc. In fact, corona extracts some of the dissipated energy inside the disc. When there is not a corona, all of the dissipated energy heats the disc. But with a corona some of the dissipated energy transfers from the disc to the corona and so, the disc becomes cooler. Generally, when the viscosity coefficient increases the disc temperature decreases both with and without a corona. In fact, the fraction of the dissipated energy into the corona is directly proportional to $\alpha_{0}$ according to equation (2). Thus, as the profile of the temperature shows, the existence of a corona leads to a cooler disc and the corona becomes more effective when the viscosity has larger values. The bottom left-hand plot of Figure 2 shows the ratio of the disc thickness to the radius. The corona causes the disc to become thinner than the case without a corona. It justifies the thin disc approximation even in the presence of a corona. The ratio of the disc thickness to the radius decreases when the viscosity coefficient increases. Hydrostatic equilibrium of the disc in the vertical direction implies that the thickness of the disc is in proportion to the sound speed 
(i.e. temperature). Since the existence of the corona reduces the temperature of the disc, larger values of the viscosity coefficient imply a thinner disc.

The bottom right-hand plot of 2 shows the ratio $\beta$ of gas pressure to the total pressure of the disc. In the absence of a corona, when the viscosity coefficient increases, the ratio of gas pressure to the total pressure decreases (i.e., radiation pressure increases). However, in a disc with a corona, the parameter $\beta$ increases as the viscosity coefficient increases (i.e., radiation pressure decreases). Generally, the presence of a corona increases the ratio of the gas pressure to the total pressure in comparison to the same disc but without a corona. Clearly, the ratio $\beta$ tends to unity at large radial distances. On the other hand, the fraction $f$ of energy exchange is directly proportional to the ratio $\beta$ (see equation (2) ). Thus, the fraction $f$ reaches to its maximum value when the ratio $\beta$ tends to the unity. Thus, the maximum value of $f$ is $\sqrt{2 \alpha_{0}}$ according to equation (21).

Figure 3 shows the typical behavior of the physical quantities of the disc for different values of the viscosity exponent $\mu$. The top left-hand plot of Figure 3 shows that the surface density of the disc increases when the viscosity exponent increases in both cases with a corona and without a corona. The presence of a corona enhances the surface density for all of the input parameters of the viscosity exponent. However, in the case of a system without a corona, the profiles are more sensitive to the variations of the viscosity exponent $\mu$. The behavior of the temperature is shown in the top righthand plot of Figure 3. The temperature of the disc increases when viscosity exponent increases for both cases with and without a corona. Generally, the presence of a corona serves to cool the disc, but as the viscosity exponent increases the disc becomes warmer in particular in the inner parts. The bottom left-hand side of Figure 3 shows that the ratio of disc thickness to the radius increases with increasing values of the parameter $\mu$, though the enhancement is not significant. The presence of a corona causes the disc to become thinner in comparison with the same disc without a corona for all of the input parameters of the viscosity exponent. The bottom right-hand plot of Figure 3 shows that the corona causes $\beta$ to increase in comparison to the case without a corona. When the viscosity exponent increases then the ratio $\beta$ increases for a disc without a corona but decreases for the case of a disc with a corona.

The measured values of the magnetic strength of the neutron stars are in the range $10^{8.5} \mathrm{G}$ to $10^{12} \mathrm{G}$ (Dewey et al 1986; Frank, King \& Raine 2002). This implies that the magnetic fields of the neutron stars in the
LMXB are at least as strong. It was shown that the magnetic field of neutron stars probably do not decay below $10^{9} \mathrm{G}$ or if they do the timescale is larger than $10^{9}$ yr (Van den Heuvel et al 1986). Typical behaviors of the physical variables with different strengths of the stellar magnetic field are shown in Figure 4. The top left-hand plot of Figure 4 shows the surface density of the disc. The surface density of the disc increases when the magnetic field increases for both cases with a corona and without a corona. The presence of a corona enhances the surface density. The top right-hand plot of Figure 4 shows the disc temperature increases when magnetic field increases for both cases with a corona and without a corona. Presence of the corona decreases temperature of the disc for all of the input parameters. Generally, we see that the corona becomes more effective when the magnetic field has larger values. The bottom left-hand plot of Figure 4 shows that the ratio of disc thickness to the radius increases when magnetic field increases. The corona causes the disc to become thinner in comparison with the same disc without a corona, also when magnetic field is stronger, the corona becomes more effective. The bottom right-hand plot of Figure 4 shows the ratio of gas pressure to the total pressure of the disc. The ratio $\beta$ decreases when the magnetic field increases for both cases with a corona and without a corona. The corona causes ratio of gas pressure to the total pressure of the disc to increase for all of the input parameters.

The effect of the spin period on the physical parameters is displayed in Figure 5 The profile of the surface density is shown in the top left-hand plot of this figure. The surface density of the disc decreases when the spin period of the star increases for both cases with a corona and without a corona. For all of the input parameters, the presence of a corona enhances the surfaces density. The right-hand plot of Figure 5 shows the corona decreases temperature of the disc in comparison with the same disc without a corona, irrespective of the spin period. Also, the temperature of the disc decreases when the spin period increases and clearly this reduction of temperature is more evident when the star is rotating slower. The bottom left-hand side of Figure 5 shows that when spin period increases then the ratio of disc thickness to the radius decreases for all cases. In this regards, the corona becomes more effective when the star is rotating faster. Also the presence of the corona causes the disc becomes thinner for all of the input parameters. The bottom right-hand plot of Figure 5 shows that the presence of a corona enhances the ratio of gas pressure to the total pressure of the disc. The ratio of gas pressure to the total pressure of the disc increases when spin period increases and even it tends 
to unity for spin periods longer than 5 seconds which implies negligible radiation pressure. Generally, we see that the corona becomes more effective when the spin period has smaller values.

\section{Conclusion}

We obtained analytic solutions for the thin accretion disc with corona, to which a magnetic torque due to the central object (e.g. neutron star) is applied. We assumed that all angular momentum transport takes place in the disc and the mass accretion rate $\dot{M}$ is constant with radius and time. In our simple model there is only energy exchange between the corona and the underlying optically thick disc. We showed that the existence of corona affects structure of the disc which is under the influence of the central magnetic field. Central in our study is the assumed existence of magnetic loops anchored in the disc and extending into a corona on both sides of the disc. Such loops are believed to arise by MRI inside the disc. We showed that the presence of a corona serves to cool the disc because of the energy transported from the disc to the corona. This reduction of the temperature causes the disc becomes thinner. The surface density increases when we consider the corona in comparison to the same disc without a corona. Our solutions indicate that the presence of corona increases ratio of the gas pressure to the total pressure.

Although we considered a generalized prescription for the viscosity, profiles of the physical variables are weakly depending on the viscosity exponent in both cases with a corona and without a corona. But behaviors of the solutions are highly depend on the viscosity coefficient. The effect of corona is more evident as viscosity tends to high values. Note that we can not directly compare our solutions with Matthews et al (2005) study. In fact, our viscosity prescription and viscosity coefficient are not similar to the analysis of Matthews et al (2005). Moreover, we considered both radiation and gas pressures. Another important factor in our model is the strength of the central magnetic field at the surface of the neutron star. For low level strength of the surface magnetic field, the effect of corona on profiles of the system is negligible. Also, in this case, the disc is in gas pressure dominated regime. Corona significantly modifies physical profiles of the system with increasing the strength of the central magnetic field. The spin period of the central neutron star is also another important factor in our model. As the star rotates faster, the effect of corona on the behaviors of the physical variables becomes more significant. These findings clearly show the importance of corona in LMXBs which can not be neglected in modeling of such systems. We note that the results are obtained based on only energy exchange from the disc to the corona. Further improvements of our model will include a more accurate description of disc-corona interactions, such as mass exchange or even angular momentum transport. Also, it will be interesting to calculate modifications to the disc spectrum due to the existence of corona according to our analytical solutions.

Acknowledgements We are grateful to the anonymous referee for very useful suggestions and comments to improve the paper. F. K. is grateful for Ad Astra PhD Scholarship of University College of Dublin. The research of M. S. was funded under the Programme for Research in Third Level Institutions (PRTLI) administered by the Irish Higher Education Authority under the National Development Plan and with partial support from the European Regional Development Fund. 


\section{References}

Aly, J. J.: A\&A, 86, 192 (1980)

Aly, J. J., Kuijpers, J.: A\&A, 227, 473 (1990)

Armitage, P. J., Clarke, C. J., Tout, C. A.: MNRAS, 304, 425 (1999)

Balbus, S. A., Hawley, J. F.: ApJ, 376, 214 (1991)

Begelman, M. C., McKee, C. F., Shields, G. A.: ApJ, 271, 89 (1983)

Burm, H.: A\&A, 165, 120 (1986)

Burm, H., Kuperus, M.: A\&A, 192, 165 (1988)

Campbell, C. G., Heptinstall, P. M.: MNRAS, 301, 558 (1998)

Church, M. J., Balucinska-Church, M.: A\&A, 300, 441 (1995)

Church, M. J., Balucinska-Church, M.: MNRAS, 348, 955 (2004)

Dai, H.-l., Li, X.-D.: A\&A, 451, 581 (2006)

Dewey, R. J., Maguire, C. M., Rawley, L. A., Stokes, G. H., Taylor, J. H.: NATUR, 322, 712 (1986)

Frank, J., King, A., Raine, D.: "Accretion Power in Astrophysics", Cambridge University Press (2002)

Ghosh, P., Lamp, F. K.: ApJ, 223, L83 (1978)

Galeev, A. A., Rosner, R., Vaiana, G. S.: ApJ, 229, 318 (1979)

Haardt, F., Maraschi, L.: ApJ, 380, L51 (1991)

Haardt, F., Maraschi, L.: ApJ, 413, 507 (1993)

Heyvaerts, J. F., Priest, E. R.: A\&A, 137, 63 (1984)

Heyvaerts, J. F., Priest, E. R.: A\&A, 216, 230 (1989)

Jimenez-Garate, M. A., Raymond, J. C., Liedahi, D. A.: ApJ, 581, 1297 (2002)

Khajenabi, F., Shadmehri, M.: MNRAS, 377, 1689 (2007)

Livio, M., Pringle, J. E.: MNRAS, 259, 23 (1992)

Matthews, O. M., Speith, R., Truss, M. R., Wynn, G. A.: MNRAS, 356, 66 (2005)

Merloni, A.: MNRAS, 341, 1051 (2003)

Merloni, A., Fabian, A. C.: MNRAS, 328, 958 (2001a)

Merloni, A., Fabian, A. C.: MNRAS, 321, 549 (2001b)

Merloni, A., Nayakshin, S.: MNRAS, 372, 728 (2006)

Miller, K. A., Stone, J. M.: APJ, 489, 890 (1997)

Romanova, M. M., Ustyugova, G. V., Koldoba, A. V., Chechetkin, V. M., Lovelace, R. V. E.: ApJ, 500, 703 (1998)

Romanova, M. M., Ustyugova, G. V., Koldoba, A. V., Lovelace, R. V. E.: ApJ, 578, 420 (2002)

Romanova, M. M., Toropina, O. D., Toropina, Y. M., Lovelace, R. V. E.: ApJ, 588, 400 (2003)

Rozanska, A., Czerny, B.: Acta Astronomica, 46, 233 (1996)

Rozanska, A., Czerny, B., Zycki, P. T., Pojmanski, G.: MNRAS, 305, 481 (1999)

Rozanska, A., Dumont, A. M., Czerny, B., Collin, S.: MNRAS, 332, 799 (2002)

Schenker, K., King, A. R., Kolb, U., Wynn, G. A., Zhang, Z.: MNRAS, 337, 110 (2002)

Shakura, N., Sunyaev, R.A.: A\&A, 24, 337 (1973)

Shibata, K., Tajima, T., Matsumoto, R.: ApJ, 350, 295 (1990)

Stella, L., Rosner, R.: ApJ, 277, 312 (1984)

Stollman, G. M., Kuperus, M.: A\&A, 203, 104 (1988)

Taam, R. E., Lin, D.N.C.: ApJ, 287, 761 (1984)
Verbunt, F.: ARA\&A, 31, 93 (1993)

Van den Heuvel, E. P. J., van Paradijs, J., Taam, R. E.: Nature, 322, 153 (1986)

Wang, Y. M.: A\&A, 183, 257 (1987)

Warner, B.: Cataclysmic Variable Stars. Camberige Univ. Press, Camberige (1995)

Watarai, K.-Y., Mineshige, S.: ApJ, 596, 421 (2003) 